\title{
Changes in the structure of a savanna forest over a six-year period in the Amazon-Cerrado transition, Mato Grosso state, Brazil
}

\author{
Mudanças na estrutura de um cerradão em um período de seis anos, \\ na transição Cerrado-Floresta Amazônica, Mato Grosso, Brasil
}

Daniel David Franczak ${ }^{1}$, Beatriz Schwantes Marimon ${ }^{2,4}$, Ben Hur Marimon-Junior ${ }^{2}$, Henrique Augusto Mews ${ }^{3}$, Leandro Maracahipes ${ }^{2}$ \& Edmar Almeida de Oliveira ${ }^{2}$

\begin{abstract}
Vegetation changes in transition zones are still poorly studied. Changes in the vegetation structure of a savanna forest (cerradão) were assessed in the Amazon-Cerrado transition (1442'2.3'S; 52²1'2.6”'W), eastern Mato Grosso, within a period of six years (2002, 2005 and 2008). In 2002, fifty plots of $10 \times 10 \mathrm{~m}$ were set up, where all trees with $\mathrm{DSH}_{30} \geq 5 \mathrm{~cm}$ were measured; in 2005 and 2008 the plots were re-inventoried. In 2008, 84 species from 70 genera and 37 families were sampled; absolute density was 1,998 individuals/ha and basal area was $25.95 \mathrm{~m}^{2} \cdot \mathrm{ha}^{-1}$. On the one hand, the absolute density of live individuals decreased from 2005 to 2008 (2,066 individuals/ha); on the other hand, the basal area increased in 2008 compared to $2005\left(23.56 \mathrm{~m}^{2} \cdot \mathrm{ha}^{-1}\right)$ and $2002\left(1,884\right.$ individuals/ha and $\left.21.38 \mathrm{~m}^{2} \cdot \mathrm{ha}^{-1}\right)$. The species with the highest importance value in the period were Hirtella glandulosa, Tachigali vulgaris and Xylopia aromatica. Except for these three species, all other species underwent hierarchic changes in the importance value, indicating that most species frequently alternate. Community structure exhibited changes throughout the period; hence, we suggest investigations on the role of T. vulgaris in these changes, since environmental conditions caused by gap opening from the fall of senile individuals of this pioneer species with a short life cycle may contribute to community dynamics.
\end{abstract}

Key words: structural changes, permanent plots, Tachigali vulgaris.

\section{Resumo}

Mudanças na vegetação em zonas de transição são ainda pouco estudadas. Foram avaliadas as mudanças na estrutura da vegetação de um cerradão na transição Cerrado-Floresta Amazônica (1442’2,3”'S e 52²1'2,6”'W), no leste de Mato Grosso, em um período de seis anos (2002, 2005 e 2008). Em 2002 foram estabelecidas 50 parcelas de $10 \times 10 \mathrm{~m}$, medidas todas as árvores com DAS $\geq 5 \mathrm{~cm}$ e em 2005 e 2008 as parcelas foram reinventariadas. Em 2008 foram amostradas 84 espécies, 70 gêneros e 37 famílias, a densidade absoluta foi de 1.998 indivíduos/ha e a área basal de $25,95 \mathrm{~m}^{2} . \mathrm{ha}^{-1}$. A densidade absoluta dos indivíduos vivos diminuiu em relação a 2005 (2.066 ind/ha); em contrapartida, a área basal aumentou em relação a $2005\left(23,56 \mathrm{~m}^{2} \cdot \mathrm{ha}^{-1}\right) \mathrm{e}$ 2002 ( $1.884 \mathrm{ind} /$ ha e $\left.21,38 \mathrm{~m}^{2} \cdot \mathrm{ha}^{-1}\right)$. As espécies com maior valor de importância nos períodos analisados foram Hirtella glandulosa, Tachigali vulgaris e Xylopia aromatica. Com exceção destas três espécies, todas as demais sofreram alterações hierárquicas no valor de importância, indicando que a maioria das espécies está se alternando frequentemente. Como a estrutura da comunidade apresentou mudanças entre os períodos estudados, sugerimos investigações sobre o papel de T. vulgaris nessas mudanças, uma vez que as condições ambientais ocasionadas pela abertura de clareiras em função da queda de indivíduos senis desta espécie pioneira e de ciclo de vida curto podem estar contribuindo na dinâmica da comunidade.

Palavras-chave: alterações estruturais, parcelas permanentes, Tachigali vulgaris.

\footnotetext{
'Universidade de Brasília - UnB, Programa de Pós-graduação em Botânica, C.P. 4457, 70.904-970, Brasília, DF, Brasil.

${ }^{2}$ Universidade do Estado de Mato Grosso - UNEMAT, Programa de Pós-graduação em Ecologia e Conservação, C. P. 08, 78.690-000, Nova Xavantina, MT, Brasil.

${ }^{3}$ Universidade de Brasília, Programa de Pós-graduação em Ciências Florestais, C. P. 4357, 70.910-900, Brasília, DF, Brasil.

${ }^{4}$ Autor para correspondência: biamarimon@hotmail.com.
} 


\section{Introduction}

The Cerrado is seen as one of the 34 biodiversity hotspots of the world (Mittermeier $e t$ al. 2005); it is the largest Neotropical savanna in the world and has the second largest area of all biomes in South America (Oliveira \& Marquis 2002). Among several threatened physiognomies of this biome, the savanna forest ("cerradão") stands out. It is denominated 'mesophyllous sclerophyllous forest' (Rizzini 1979) and is characterized by the presence of species from savanna and forest environments. In general, this physiognomy is not tolerant of anthropic disturbance and occupies small areas; exactly the areas that are most frequently used for agriculture and livestock, since these areas are usually on soils with higher availability of exchangeable cations (Ratter 1971; Eiten 1979; Oliveira-Filho et al. 1994), i.e. more humid and with clayey texture (Marimon-Junior \& Haridasan 2005).

In the eastern portion of Mato Grosso state there are patches of savanna forest in the transition zone between Cerrado and the Amazon biomes (Marimon-Junior \& Haridasan 2005; Marimon et al. 2006). Two types of vegetation were recorded in the area: the savanna forest of Magonia pubescens A. St.-Hil. and Callisthene fasciculata Mart., which occurs on mesotrophic soils; and the savanna forest of Hirtella glandulosa, which occurs on dystrophic soils (Ratter 1971; Ratter et al. 1973). These two savanna forest types are threatened, mainly because they are located in a region known as the 'deforestation arch', where the advance of cultivated areas represents an important threat to native vegetation (Nogueira et al. 2008).

Most studies carried out in savanna forests are based on information collected at a point in time (Costa \& Araújo 2001; Marimon \& Lima 2001; Gomes et al. 2004; Marimon-Junior \& Haridasan 2005; Guilherme \& Nakajima 2007; Kunz et al. 2009). There is still a huge need for studies on the dynamics of this vegetation. Therefore, studies on long-term vegetation changes are essential to understand the mechanisms and processes that maintain the community in a steady state (Aquino $e t$ al. 2007). A lot of information obtained from nativeforest functioning can be used for its management.

Hence, important subsidies to practices of conservation, management and restoration of degraded areas, for example, may come from studies on the remnants of native vegetation in Mato Grosso state. Therefore, the objective of this study was to assess changes in the structure of the woody vegetation of a savanna forest in eastern Mato Grosso, in the transition region between Cerrado and the Amazon, within a period of six years (2002 and 2008). The transition between Cerrado and the Amazon extends for over 4,500 km (Ackerly et al. 1989) and it is dynamic: studies show that forests are advancing over savannas (Marimon et al. 2006). In this context, the present study will also loot at whether floristic and structural changes in the savanna forest led this physiognomy to become a denser forest.

\section{Material and Methods}

The study was carried out in a savanna forest (14²' 2.3”'s; 52²1'2.6”W), Bacaba Municipal Park, Nova Xavantina, state of Mato Grosso, centralwestern Brazil. According to Marimon-Junior \& Haridasan (2005), the park is located in a transition region between the Cerrado and Amazon biomes, where the predominant 'cerrado sensu stricto' vegetation (open savanna) is in contact with forests and savanna forests, in acid and dystrophic soils, with high levels of exchangeable aluminum and clayey texture. According to Köppen's classification, the regional climate is type Aw (Silva et al. 2008), characterized by two well-defined seasons: one dry and cold (April to September) and the other hot and rainy (October to March).

The transition between Cerrado and the Amazon is a zone of ecological tension that exhibits a mosaic of savannas and forests (Ratter et al. 1973; Ackerly et al. 1989; Ivanauskas et al. 2004; Marimon et al. 2006). Based on IBGE (2004), our study area is about $150 \mathrm{~km}$ from this zone of ecological tension. However, this zone is not regular; there are larger or smaller intrusions or fringes (Ratter et al. 1973; Marimon et al. 2006), which are currently fragmented due to the conversion of native vegetation into agricultural lands (Nogueira et al. 2008). Although our study area is located in a vegetation matrix dominated by savanna (Marimon-Junior \& Haridasan 2005), in nearby areas (less than $10 \mathrm{~km}$ ) there are fragments and intrusions of contact between savanna and seasonal forest (IBGE 2004; Marimon 2005). Records taken out in 1943 by members of the Roncador-Xingu Expedition confirmed that up to 40 $\mathrm{km}$ to the south of our study area there would have existed 'a dense vegetation, where to open the way they needed to cut down colossal trees' (Carpentieri 2008); this report characterizes the study region as a zone of ecological tension or a zone of transition between Cerrado and the Amazon. 
In 2002 , fifty $10 \times 10 \mathrm{~m}$ permanent plots were set up (Marimon-Junior \& Haridasan 2005), where woody species (except lianas) that had $\mathrm{DSH}_{30}$ (diameter at soil height, measured at $30 \mathrm{~cm}$ ) e $\geq 5 \mathrm{~cm}$ were sampled. At the occasion, all individuals were tagged with numbered aluminum plates, and were recorded and identified. Species were identified by comparison with herbaria vouchers (NX and UB) and by consulting specialists. The collected material was deposited in the NX Herbarium, UNEMAT Nova Xavantina Campus, Mato Grosso state. In 2005 and in 2008, all surviving individuals were measured again and recruits (individuals that reached the minimal inclusion criterion) were tagged, measured, recorded and identified. Sampling and species identification followed the same procedures used in the first inventory. The classification system used for families was APG III (2009) and the revision of taxa names followed Forzza et al. (2010) in the list of Angiosperm species of the Brazilian flora.

Parameters of relative density, frequency, dominance and importance value (IV) were used to describe vegetation structure, following MüellerDombois \& Ellenberg (1974). The program FITOPAC 1.0 (Shepherd 1994) was used for the analysis. We compared the phytosociology of the three inventories: 2002 (Marimon-Junior \& Haridasan 2005), 2005 and 2008 (present study).

Based on the number of individuals sampled, mortality and recruitment rates were calculated for each plot (Sheil et al. 1995, 2000) and comparisons were made (2002-2005 and 2005-2008) using paired $t$-tests. The average number of individuals and the basal area in each year sampled were compared by analysis of variance and Tukey's test at 5\% probability (Zar 1999).

\section{Results and Discussion}

In 2008, the savanna forest studied had 84 plant species from 70 genera and 37 families (Tab. 1), with an absolute density of 1,998 individuals/ha and a basal area of $25.95 \mathrm{~m}^{2}$.ha-1. On the one hand, the absolute density of live individuals decreased compared to 2005 (2,066 ind.ha $\left.{ }^{-1}\right)$; on the other hand, the basal area increased compared to 2002 and 2005 (Tab. 2). In 2002, 77 species from 65 genera and 36 families were recorded (Marimon-Junior \& Haridasan 2005) and in 2005, 87 species from 71 genera and 38 families. The increase in the basal area in two consecutive periods (2005 and 2008) in the savanna forest is consistent with Phillips et al.
(2002), Baker et al. (2004) and Lewis et al. (2009), who observed that in the last century nearly all terrestrial ecosystems have been under the influence of atmospheric and climatic changes. An increase in dynamics, biomass and carbon stock in tropical forests was recorded, probably due to an increase of $\mathrm{CO}_{2}$ levels in the atmosphere. Further detailed long-term studies in the savanna forest studied here are essential to verify if the increase in biomass of this community is related to the increase in atmospheric $\mathrm{CO}_{2}$ levels. However, the increase in biomass could also be explained by climatic changes that have been occurring since the early Holocene, when a drier climate was replaced by a warmer and more humid climate (Ledru et al. 1996). In a study carried out by Marimon et al. (2006) in a nearby area, 30-year records showed that the Amazon advanced $7 \mathrm{~km}$ into the Cerrado, reinforcing the expansion of forests over savannas in the region.

Recruitment and mortality rates were higher in the first inventory period, between 2002 and 2005 (Tab. 2). If we consider intervals as well as the whole period (2002 to 2008), the values of the savanna forest of Bacaba Park were higher than the values observed in other studies carried out in forests of South and Central America, which varied from 0.5 to $2.8 \%$.year $^{-1}$ for mortality (Lieberman et al. 1985 ; Swaine et al. 1987; Condit et al. 1995; Felfili 1995) and from 2 to $4 \%$.year $^{-1}$ for recruitment (OliveiraFilho et al. 1997; Higuchi et al. 2008; Silva \& Araújo 2009; Miguel et al. 2011). According to Felfili (1995), mortality rates around $3.5 \%$.year $^{-1}$ are typical of areas that underwent disturbances. Oliveira \& Felfili (2008) observed that high mortality and recruitment rates lead to a high turnover, confirming the dynamic aspect of the community, which even without undertaking direct disturbances (fire and cutting) exhibited high mortality and recruitment. Considering the whole period (2002-2008), recruitment compensated mortality $(t=-2.95, P=$ $0.0024)$. This compensation can be related to a 'construction' phase of the sylvigenetic cycle of the community, as proposed by Hallé et al. (1978), which is usually recorded in forests recovering from a disturbance (Oliveira-Filho et al. 1997; Chagas et al. 2001), suggesting that periods of higher mortality might have previously occurred (Felfili 1995), of which there is no record from the memory of local residents.

The reduction in density and the increase in basal area recorded in the present study (Tab. 2) are consistent with a self-thinning pattern, as observed by Felfili (1995) and Werneck et al. (2000). 
Table 1 - Phytosociological parameters of species sampled in a cerradão in the Cerrado-Amazon Forest transition, in 2005 and 2008, Nova Xavantina-MT. N ${ }^{\circ}$ Herb.= registration number in Herbarium NX, N= number of individuals, $\mathrm{DR}=$ relative density $(\%), \mathrm{FR}=$ relative frequency $(\%)$, DoR= relative dominance $(\%)$, and $\mathrm{VI}=$ importance value. Species listed in order of decreasing VI.

\begin{tabular}{|c|c|c|c|c|c|c|c|c|c|c|c|c|}
\hline \multirow[t]{2}{*}{ Species } & \multirow{2}{*}{ Families } & \multirow{2}{*}{$\begin{array}{l}\mathbf{N}^{\mathbf{o}} \\
\text { Herb. }\end{array}$} & \multicolumn{2}{|c|}{$\mathbf{N}$} & \multicolumn{2}{|c|}{ DR } & \multicolumn{2}{|c|}{ FR } & \multicolumn{2}{|c|}{ DoR } & \multicolumn{2}{|c|}{ VI } \\
\hline & & & 2005 & 2008 & 2005 & 2008 & 2005 & 2008 & 2005 & 2008 & 2005 & 2008 \\
\hline Hirtella glandulosa Spreng. & Chrysobalanaceae & 728 & 120 & 125 & 11,62 & 12,52 & 6,12 & 6,48 & 25,17 & 27,37 & 42,90 & 46,37 \\
\hline Tachigali vulgaris L.G.Silva \& H.C.Lima & Fabaceae & 674 & 85 & 93 & 8,23 & 9,31 & 5,12 & 4,86 & 10,95 & 11,21 & 24,30 & 25,38 \\
\hline Xylopia aromatica $(\mathrm{Lam}$.$) Mart.$ & Annonaceae & 137 & 74 & 63 & 7,16 & 6,31 & 5,55 & 5,15 & 7,55 & 7,00 & 20,26 & 18,46 \\
\hline Tapirira guianensis Aubl. & Anacardiaceae & 59 & 39 & 42 & 3,78 & 4,20 & 3,70 & 3,98 & 2,95 & 3,86 & 10,42 & 12,04 \\
\hline Emmotum nitens (Benth.) Miers & Icacinaceae & 1371 & 22 & 22 & 2,13 & 2,20 & 2,42 & 2,50 & 6,09 & 6,48 & 10,64 & 11,18 \\
\hline Myrcia splendens (Sw.) DC & Myrtaceae & 2258 & 41 & 41 & 3,97 & 4,10 & 3,70 & 3,83 & 2,64 & 2,73 & 10,31 & 10,66 \\
\hline Chaetocarpus echinocarpus (Baill.) Ducke & Peraceae & 1046 & 41 & 42 & 3,97 & 4,20 & 3,84 & 3,98 & 1,96 & 2,21 & 9,77 & 10,39 \\
\hline Matayba guianensis Aubl. & Sapindaceae & 9518 & 33 & 35 & 3,19 & 3,50 & 3,41 & 3,83 & 2,04 & 2,15 & 8,65 & 9,48 \\
\hline Heisteria ovata Benth. & Olacaceae & 2403 & 44 & 41 & 4,26 & 4,10 & 3,70 & 3,68 & 1,67 & 1,62 & 9,63 & 9,40 \\
\hline Aspidosperma multiflorum A.DC. & Apocynaceae & 164 & 33 & 32 & 3,19 & 3,20 & 3,41 & 3,39 & 2,35 & 2,20 & 8,96 & 8,79 \\
\hline Vatairea macrocarpa (Benth.) Ducke & Fabaceae & 1275 & 27 & 26 & 2,61 & 2,60 & 2,99 & 3,39 & 2,27 & 2,35 & 7,87 & 8,34 \\
\hline Eriotheca gracilipes (K. Schum.) A.Robyns & Malvaceae & 477 & 25 & 21 & 2,42 & 2,10 & 2,28 & 2,21 & 4,50 & 3,08 & 9,19 & 7,39 \\
\hline Sorocea klotzschiana Baill. & Moraceae & 2117 & 28 & 31 & 2,71 & 3,10 & 2,56 & 2,80 & 0,96 & 1,27 & 6,23 & 7,17 \\
\hline Guapira graciliflora (Mart. ex Schmidt) Lundell & Nyctaginaceae & 2322 & 33 & 26 & 3,19 & 2,60 & 3,27 & 2,80 & 1,37 & 1,17 & 7,84 & 6,57 \\
\hline Roupala montana Aubl. & Proteaceae & 2493 & 32 & 22 & 3,10 & 2,20 & 3,41 & 2,50 & 2,66 & 1,51 & 9,17 & 6,22 \\
\hline Alchornea discolor Poepp. & Euphorbiaceae & 1032 & 15 & 17 & 1,45 & 1,70 & 1,99 & 2,36 & 0,85 & 0,93 & 4,29 & 4,99 \\
\hline Siparuna guianensis Aubl. & Siparunaceae & 2075 & 18 & 20 & 1,74 & 2,00 & 1,99 & 2,21 & 0,61 & 0,77 & 4,34 & 4,98 \\
\hline Maprounea guianensis Aubl. & Euphorbiaceae & 1103 & 14 & 15 & 1,36 & 1,50 & 1,71 & 1,91 & 0,81 & 1,08 & 3,87 & 4,50 \\
\hline Cordiera sessilis (Vell.) Kuntze & Rubiaceae & 2526 & 16 & 16 & 1,55 & 1,60 & 2,13 & 2,21 & 0,54 & 0,52 & 4,22 & 4,33 \\
\hline Pseudobombax longiflorum (Mart.\& Zucc.)A.Robyns & Malvaceae & 484 & 14 & 14 & 1,36 & 1,40 & 1,56 & 1,62 & 0,95 & 0,93 & 3,87 & 3,95 \\
\hline Agonandra brasiliensis Miers ex Benth. \& Hook. f. & Opiliaceae & 2422 & 9 & 12 & 0,87 & 1,20 & 1,00 & 1,33 & 0,81 & 0,87 & 2,68 & 3,39 \\
\hline Erythroxylum daphnites Mart. & Erythroxylaceae & 6980 & 17 & 13 & 1,65 & 1,30 & 1,85 & 1,62 & 0,49 & 0,30 & 3,99 & 3,22 \\
\hline Terminalia argentea Mart. & Combretaceae & 846 & 10 & 9 & 0,97 & 0,90 & 1,28 & 1,33 & 1,02 & 0,84 & 3,27 & 3,07 \\
\hline Protium heptaphyllum (Aubl.) Marchand & Burseraceae & 519 & 8 & 8 & 0,77 & 0,80 & 1,00 & 1,03 & 0,83 & 0,98 & 2,60 & 2,81 \\
\hline Buchenavia tomentosa Eichler & Combretaceae & 833 & 6 & 6 & 0,58 & 0,60 & 0,85 & 0,88 & 1,02 & 1,29 & 2,46 & 2,78 \\
\hline Alibertia edulis (Rich.) A.Rich. & Rubiaceae & 2513 & 9 & 10 & 0,87 & 1,00 & 1,28 & 1,47 & 0,23 & 0,30 & 2,39 & 2,77 \\
\hline Lacistema aggregatum (P.J.Bergius) Rusby & Lacistemataceae & 1382 & 9 & 11 & 0,87 & 1,10 & 1,14 & 1,33 & 0,19 & 0,24 & 2,19 & 2,67 \\
\hline Pterodon pubescens (Benth.) Benth. & Fabaceae & 6975 & 6 & 6 & 0,58 & 0,60 & 0,71 & 0,74 & 1,25 & 1,24 & 2,55 & 2,57 \\
\hline
\end{tabular}




\begin{tabular}{|c|c|c|c|c|c|c|c|c|c|c|c|c|}
\hline \multirow[t]{2}{*}{ Species } & \multirow[t]{2}{*}{ Families } & \multirow{2}{*}{$\begin{array}{l}\mathbf{N}^{\mathbf{o}} \\
\text { Herb. }\end{array}$} & \multicolumn{2}{|c|}{$\mathbf{N}$} & \multicolumn{2}{|c|}{ DR } & \multicolumn{2}{|c|}{ FR } & \multicolumn{2}{|c|}{ DoR } & \multicolumn{2}{|c|}{ VI } \\
\hline & & & 2005 & 2008 & 2005 & 2008 & 2005 & 2008 & 2005 & 2008 & 2005 & 2008 \\
\hline Vochysia haenkeana Mart. & Vochysiaceae & 3066 & 6 & 7 & 0,58 & 0,70 & 0,85 & 0,88 & 0,68 & 0,92 & 2,11 & 2,51 \\
\hline Aspidosperma macrocarpon Mart. & Apocynaceae & 158 & 8 & 8 & 0,77 & 0,80 & 1,14 & 1,18 & 0,46 & 0,43 & 2,37 & 2,41 \\
\hline Mezilaurus crassiramea (Meisn.) Taub. ex Mez & Lauraceae & 1463 & 4 & 4 & 0,39 & 0,40 & 0,57 & 0,59 & 1,54 & 1,38 & 2,49 & 2,37 \\
\hline Antonia ovata Pohl & Loganiaceae & 1523 & 14 & 9 & 1,36 & 0,90 & 1,71 & 1,18 & 0,42 & 0,28 & 3,49 & 2,36 \\
\hline Coccoloba mollis Casar. & Polygonaceae & 9522 & 9 & 9 & 0,87 & 0,90 & 0,85 & 0,88 & 0,48 & 0,65 & 2,21 & 2,35 \\
\hline Salvertia convallariodora A.St.-Hil. & Vochysiaceae & 3057 & 8 & 9 & 0,77 & 0,90 & 0,85 & 1,03 & 0,27 & 0,25 & 1,89 & 2,18 \\
\hline Qualea parviflora Mart. & Vochysiaceae & 3044 & 8 & 8 & 0,77 & 0,80 & 1,00 & 1,03 & 0,37 & 0,34 & 2,14 & 2,17 \\
\hline Curatella americana $\mathrm{L}$. & Dilleniaceae & 927 & 5 & 5 & 0,48 & 0,51 & 0,71 & 0,74 & 0,95 & 0,91 & 2,15 & 2,14 \\
\hline Luetzelburgia praecox (Harms) Harms & Fabaceae & 1235 & 6 & 6 & 0,58 & 0,60 & 0,85 & 0,88 & 0,60 & 0,58 & 2,04 & 2,06 \\
\hline Dipteryx alata Vogel & Fabaceae & 1219 & 4 & 4 & 0,39 & 0,40 & 0,57 & 0,59 & 0,86 & 1,01 & 1,81 & 2,00 \\
\hline Syagrus comosa (Mart.) Mart. & Arecaceae & 3112 & 8 & 7 & 0,77 & 0,70 & 0,85 & 0,88 & 0,45 & 0,33 & 2,08 & 1,92 \\
\hline Guapira noxia (Netto) Lundell & Nyctaginaceae & 2324 & 10 & 6 & 0,97 & 0,60 & 1,00 & 0,74 & 0,76 & 0,56 & 2,73 & 1,90 \\
\hline Qualea grandiflora Mart. & Vochysiaceae & 3001 & 7 & 6 & 0,68 & 0,60 & 1,00 & 0,88 & 0,44 & 0,36 & 2,11 & 1,84 \\
\hline Astronium fraxinifolium Schott & Anacardiaceae & 49 & 6 & 6 & 0,58 & 0,60 & 0,71 & 0,74 & 0,47 & 0,44 & 1,76 & 1,78 \\
\hline Syagrus flexuosa (Mart.) Becc. & Arecaceae & 3111 & 12 & 6 & 1,16 & 0,60 & 1,14 & 0,88 & 0,33 & 0,18 & 2,63 & 1,66 \\
\hline Tabebuia aurea (Silva Manso) & Bignoniaceae & 458 & 5 & 5 & 0,48 & 0,50 & 0,71 & 0,74 & 0,20 & 0,19 & 1,39 & 1,42 \\
\hline Benth \& Hook f. ex S. Moore & & & & & & & & & & & & \\
\hline Copaifera langsdorffii Desf. & Fabaceae & 580 & 3 & 4 & 0,29 & 0,40 & 0,43 & 0,59 & 0,26 & 0,36 & 0,98 & 1,35 \\
\hline Strychnos pseudoquina A.St.-Hil. & Loganiaceae & 1526 & 2 & 2 & 0,19 & 0,20 & 0,28 & 0,29 & 0,94 & 0,70 & 1,42 & 1,19 \\
\hline Mimosa laticifera Rizzini \& A.Mattos & Fabaceae & 2032 & 5 & 4 & 0,48 & 0,40 & 0,57 & 0,59 & 0,16 & 0,12 & 1,21 & 1,11 \\
\hline Ouratea spectabilis (Mart.) Engl. & Ochnaceae & 2372 & 5 & 4 & 0,48 & 0,40 & 0,71 & 0,59 & 0,14 & 0,10 & 1,34 & 1,09 \\
\hline Euplassa inaequalis (Pohl) Engl. & Proteaceae & 2478 & 4 & 3 & 0,39 & 0,30 & 0,57 & 0,44 & 0,58 & 0,33 & 1,53 & 1,07 \\
\hline Brosimum gaudichaudii Trécul & Moraceae & 2083 & 4 & 4 & 0,39 & 0,40 & 0,43 & 0,44 & 0,16 & 0,14 & 0,97 & 0,98 \\
\hline Annona coriacea Mart. & Annonaceae & 76 & 6 & 3 & 0,58 & 0,30 & 0,71 & 0,44 & 0,35 & 0,17 & 1,65 & 0,91 \\
\hline Machaerium acutifolium (Vogel) & Fabaceae & 1238 & 3 & 3 & 0,29 & 0,30 & 0,43 & 0,44 & 0,13 & 0,13 & 0,85 & 0,88 \\
\hline Rudgea viburnoides (Cham.) Benth. & Rubiaceae & 2623 & 3 & 3 & 0,29 & 0,30 & 0,43 & 0,44 & 0,07 & 0,08 & 0,79 & 0,82 \\
\hline Magonia pubescens A.St.-Hil & Sapindaceae & 2676 & 2 & 2 & 0,19 & 0,20 & 0,28 & 0,29 & 0,30 & 0,31 & 0,78 & 0,81 \\
\hline Vochysia rufa Mart. & Vochysiaceae & 3083 & 2 & 2 & 0,19 & 0,20 & 0,28 & 0,29 & 0,27 & 0,26 & 0,75 & 0,75 \\
\hline Byrsonima coccolobifolia Kunth & Malpighiaceae & 1618 & 2 & 2 & 0,19 & 0,20 & 0,28 & 0,29 & 0,28 & 0,25 & 0,75 & 0,75 \\
\hline Hymenaea stigonocarpa Mart. ex Hayne & Fabaceae & 614 & 3 & 3 & 0,29 & 0,30 & 0,28 & 0,29 & 0,10 & 0,10 & 0,68 & 0,70 \\
\hline Ficus sp. & Moraceae & 4034 & 1 & 2 & 0,10 & 0,20 & 0,14 & 0,29 & 0,03 & 0,12 & 0,27 & 0,61 \\
\hline
\end{tabular}




\begin{tabular}{|c|c|c|c|c|c|c|c|c|c|c|c|c|}
\hline \multirow[t]{2}{*}{ Species } & \multirow[t]{2}{*}{ Families } & \multirow{2}{*}{$\begin{array}{l}\mathbf{N}^{\mathbf{0}} \\
\text { Herb. }\end{array}$} & \multicolumn{2}{|c|}{$\mathbf{N}$} & \multicolumn{2}{|c|}{ DR } & \multicolumn{2}{|c|}{ FR } & \multicolumn{2}{|c|}{ DoR } & \multicolumn{2}{|c|}{ VI } \\
\hline & & & 2005 & 2008 & 2005 & 2008 & 2005 & 2008 & 2005 & 2008 & 2005 & 2008 \\
\hline Platypodium elegans Vogel & Fabaceae & 1250 & 1 & 1 & 0,10 & 0,10 & 0,14 & 0,15 & 0,32 & 0,33 & 0,56 & 0,58 \\
\hline Tapura amazonica Poepp. \& Endl. & Dichapetalaceae & 900 & 2 & 2 & 0,19 & 0,20 & 0,28 & 0,29 & 0,07 & 0,08 & 0,55 & 0,57 \\
\hline Couepia grandiflora (Mart. \& Zucc.) Benth. & Chrysobalanaceae & 718 & 2 & 2 & 0,19 & 0,20 & 0,28 & 0,29 & 0,08 & 0,07 & 0,56 & 0,57 \\
\hline Styrax camporum Pohl & Styracaceae & 2891 & 3 & 2 & 0,29 & 0,20 & 0,43 & 0,29 & 0,31 & 0,07 & 1,03 & 0,57 \\
\hline Qualea multiflora Mart. & Vochysiaceae & 3040 & 3 & 2 & 0,29 & 0,20 & 0,43 & 0,29 & 0,08 & 0,06 & 0,80 & 0,56 \\
\hline Plathymenia reticulata Benth. & Fabaceae & 2059 & 2 & 2 & 0,19 & 0,20 & 0,28 & 0,29 & 0,07 & 0,06 & 0,54 & 0,55 \\
\hline Ouratea hexasperma (A.St.-Hil.) Baill. & Ochnaceae & 2361 & 2 & 2 & 0,19 & 0,20 & 0,28 & 0,29 & 0,04 & 0,04 & 0,52 & 0,53 \\
\hline Eugenia gemmiflora O.Berg & Myrtaceae & 2270 & 2 & 2 & 0,19 & 0,20 & 0,28 & 0,29 & 0,05 & 0,03 & 0,53 & 0,53 \\
\hline Licania humilis Cham. \& Schltdl. & Chrysobalanaceae & 3563 & 1 & 2 & 0,10 & 0,20 & 0,14 & 0,29 & 0,02 & 0,03 & 0,26 & 0,53 \\
\hline Aspidosperma subincanum Mart. & Apocynaceae & 174 & 1 & 1 & 0,10 & 0,15 & 0,14 & 0,16 & 0,24 & 0,25 & 0,48 & 0,49 \\
\hline Coccoloba sp. & Polygonaceae & 9830 & 1 & 1 & 0,10 & 0,10 & 0,14 & 0,15 & 0,06 & 0,09 & 0,30 & 0,34 \\
\hline Peltogyne confertiflora (Mart. ex Hayne) Benth. & Fabaceae & 634 & 1 & 1 & 0,10 & 0,10 & 0,14 & 0,15 & 0,06 & 0,06 & 0,30 & 0,31 \\
\hline Bowdichia virgilioides Kunth & Fabaceae & 1166 & 1 & 1 & 0,10 & 0,10 & 0,14 & 0,15 & 0,06 & 0,05 & 0,30 & 0,30 \\
\hline Cybistax antisiphilitica (Mart.) Mart. & Bignoniaceae & 420 & 1 & 1 & 0,10 & 0,10 & 0,14 & 0,15 & 0,08 & 0,05 & 0,32 & 0,30 \\
\hline Andira vermifuga (Mart.) Benth. & Fabaceae & 1159 & 1 & 1 & 0,10 & 0,10 & 0,14 & 0,15 & 0,05 & 0,05 & 0,29 & 0,30 \\
\hline Polygalaceae - N.I. & Polygalaceae & 1533 & 1 & 1 & 0,10 & 0,10 & 0,14 & 0,15 & 0,04 & 0,05 & 0,28 & 0,30 \\
\hline Pterodon emarginatus Vogel & Fabaceae & 1260 & 1 & 1 & 0,10 & 0,10 & 0,14 & 0,15 & 0,04 & 0,04 & 0,27 & 0,29 \\
\hline Ficus enormis Mart. ex Miq. & Moraceae & 2090 & 1 & 1 & 0,10 & 0,10 & 0,14 & 0,15 & 0,03 & 0,02 & 0,27 & 0,28 \\
\hline Pouteria aff. gardneri (Mart. \& Miq.) Baehni & Sapotaceae & 2719 & 1 & 1 & 0,10 & 0,10 & 0,14 & 0,15 & 0,02 & 0,02 & 0,26 & 0,27 \\
\hline Byrsonima basiloba A.Juss. & Malpighiaceae & 1615 & 1 & 1 & 0,10 & 0,10 & 0,14 & 0,15 & 0,03 & 0,01 & 0,27 & 0,27 \\
\hline Cardiopetalum calophyllum Schltdl. & Annonaceae & 95 & 2 & 1 & 0,19 & 0,10 & 0,28 & 0,15 & 0,06 & 0,01 & 0,53 & 0,27 \\
\hline Dalbergia miscolobium Benth. & Fabaceae & 1196 & 1 & 1 & 0,10 & 0,10 & 0,14 & 0,15 & 0,03 & 0,01 & 0,27 & 0,27 \\
\hline Diospyros sericea A.DC. & Ebenaceae & 953 & 1 & 1 & 0,10 & 0,10 & 0,14 & 0,15 & 0,02 & 0,01 & 0,26 & 0,27 \\
\hline Miconia albicans (Sw.) Triana & Melastomataceae & 6913 & 2 & 1 & 0,19 & 0,10 & 0,28 & 0,15 & 0,07 & 0,01 & 0,55 & 0,27 \\
\hline Andira cujabensis Benth. & Fabaceae & 1156 & 1 & 1 & 0,10 & 0,10 & 0,14 & 0,15 & 0,02 & 0,01 & 0,26 & 0,26 \\
\hline Aspidosperma nobile Müll.Arg. & Apocynaceae & 173 & 1 & 1 & 0,10 & 0,10 & 0,14 & 0,15 & 0,02 & 0,01 & 0,26 & 0,26 \\
\hline Simarouba versicolor A. St.-Hil. & Simaroubaceae & 6646 & 1 & - & 0,10 & - & 0,17 & - & 0,21 & - & 0,43 & - \\
\hline Dimorphandra mollis Benth. & Fabaceae & 598 & 1 & - & 0,10 & - & 0,17 & - & 0,02 & - & 0,26 & - \\
\hline Cordiera elliptica (Cham.) Kuntze & Rubiaceae & 6825 & 1 & - & 0,10 & - & 0,16 & - & 0,02 & - & 0,26 & - \\
\hline Total & & & 1033 & 999 & 100 & 100 & 100 & 100 & 100 & 100 & 300 & 300 \\
\hline
\end{tabular}

Platypodium elegans Voge

Plathymenia reticulata Benth.

Eugenia gemmiflora O.Berg

Peltogyne confertiflora (Mart. ex Hayne) Benth.

Cybistax antisiphilitica (Mart.) Mart.

Pterodon emarginatus Vogel

Pouteria aff. gardneri (Mart. \& Miq.) Baehni

Cardiopetalum calophyllum Schltdl. 
Table 2 - Parameters of dynamics in a woody community of a cerradão in Bacaba Park, Nova Xavantina, Mato Grosso, between 2002 and 2005, 2005 and 2008, and 2002 and 2008. Where: $t=$ paired $t$-test, $\mathrm{F}=$ result of analysis of variance. Values in parentheses correspond to standard deviation. Different letters indicate differences at $5 \%$ significance level.

\begin{tabular}{|c|c|c|c|c|}
\hline Parameters & 2002 & 2005 & 2008 & \\
\hline \multirow[t]{3}{*}{ Mortality rate $\left(\%\right.$.year $\left.{ }^{-1}\right)$} & 5,52 & \multirow{2}{*}{\multicolumn{2}{|c|}{2,70}} & \multirow{3}{*}{$\begin{array}{l}t=3,62 \\
P=0,0003\end{array}$} \\
\hline & & & & \\
\hline & & 4,51 & & \\
\hline \multirow[t]{3}{*}{ Recruitment rate $\left(\%\right.$.year $\left.{ }^{-1}\right)$} & 8,47 & \multirow{2}{*}{\multicolumn{2}{|c|}{2,00}} & \multirow{3}{*}{$\begin{array}{l}t=9,67 \\
P<0,0001\end{array}$} \\
\hline & & & & \\
\hline & & \multicolumn{2}{|l|}{5,87} & \\
\hline Average number of individuals (plot) & $18,8(5,6)$ & $20,7(5,7)$ & $19,9(5,4)$ & $\begin{array}{l}\mathrm{F}=1,33 \\
P=0,2658\end{array}$ \\
\hline Basal área $\left(\mathrm{m}^{2} \cdot \mathrm{ha}^{-1}\right)$ & $21,38^{\mathrm{a}}(5,9)$ & $23,56^{a}(7,5)$ & $25,95^{\mathrm{b}}(8,4)$ & $\begin{array}{l}\mathrm{F}=4,78 \\
P=0,0098\end{array}$ \\
\hline
\end{tabular}

In a riparian forest in Distrito Federal, Oliveira \& Felfili (2005) recorded intense reduction in total density and increase in basal area; they suggested that greater shading of the area would hinder the growth of heliophilous species. This could also be happening in our study area, since all species that entered the community between 2002 and 2008, such as Diospyros sericea, Ficus enormis and Pouteria gardneri, are typical of forests and the ones that left the community, such as Dimorphandra mollis, Cordiera elliptica and Simarouba versicolor, are typical of savanna formations and open environments (Tab. 1, Marimon-Junior \& Haridasan 2005). Besides, of the eight species that exhibited higher recruitment ( $>10$ individuals) in the period from 2002 to 2008 (Tab. 3), at least six are typical of forests; and all species that exhibited higher mortality (>10 individuals, Tab. 3) are typical of savanna and field vegetation (Ratter et al. 1973; Pott \& Pott 1994; Oliveira-Filho \& Ratter 1995; IBGE 2002; Durigan et al. 2004; Mendonça et al. 2008).

The ten most important species in 2008 represented c. 54\% of the total importance value (IV) and of the total number of individuals sampled. In 2002 they represented $53 \%$ of IV and $51.7 \%$ of the total number of individuals sampled, and in $2005,52 \%$ of IV and $51.5 \%$ of the total number of individuals (Table 1; Marimon-Junior \& Haridasan 2005). In a riparian forest in Distrito Federal, Felfili (1993) observed that the ten most important species might be considered to be the ones that exhibit higher success exploiting resources of the habitat. Inventories carried out in forests and savannas of the Cerrado biome (Costa \& Araújo 2001; Marimon et al. 2006; Kunz et al. 2009) reported that the species that have higher importance value also have higher number of individuals, as recorded in the present study.

The most important species (IV) in all three sampling periods was Hirtella glandulosa, which contributed with approximately $12 \%$ of the total number of individuals in 2002, 2005 and 2008, confirming the area as a savanna forest of Hirtella glandulosa, as described by Ratter (1971) and Ratter et al. (1973). Tachigali vulgaris (=Sclerolobium paniculatum) was the second most important species in all inventories, with $6.5 \%$ of the total number of individuals in 2002 (Marimon-Junior \& Haridasan 2005), 8.2\% in 2005 and $9.3 \%$ in 2008. Xylopia aromatica was the third most important species in all three inventories, with $7.5 \%$ of the total number of individuals in 2002 (Marimon-Junior \& Haridasan 2005), 7.2\% in 2005 and 6.3\% in 2008. The species mentioned were also among the ten most important species in other savanna forest areas (Gomes et al. 2004; Pereira-Silva et al. 2004; Marimon et al. 2006; Guilherme \& Nakajima 2007; Kunz et al. 2009) and in a dense savanna (Andrade $e t$ al. 2002), evidencing their broad distribution and high importance in different forests of the Cerrado biome.

Tachigali vulgaris has a short life cycle $(<20$ years) and rapid growth (Felfili et al. 1999). In this case, it is suggested that the mortality of this species and the resulting fall of large-sized senile individuals, such as recorded by Franczak (2009) in 
Table 3 - Number of dead and recruited individuals in the intervals between the years 2002 and 2005 (02-05), 2005 and 2008 (05-08), and 2002 and 2008 (02-08). Cerradão of the Bacaba Park, Nova Xavantina, MT. Species listed in descending order of number of individuals recruited between 2002 and 2008. Were considered only those species that presented at least five dead or recruited individuals at least in one interval.

\begin{tabular}{lcccccc}
\hline Species & (02-05) & $\begin{array}{c}\text { Dead } \\
\text { (02-05) }\end{array}$ & $\mathbf{( 0 2 - 0 8 )}$ & (02-05) & $\begin{array}{c}\text { Recruited } \\
\text { (05-08) }\end{array}$ & (02-08) \\
\hline Tachigali vulgaris & 12 & 8 & 20 & 36 & 17 & 53 \\
Hirtella glandulosa & 5 & 0 & 5 & 14 & 5 & 19 \\
Heisteria ovata & 1 & 2 & 3 & 18 & - & 18 \\
Sorocea klotzschiana & - & - & - & 15 & 3 & 18 \\
Tapirira guianensis & 1 & - & 1 & 15 & 3 & 18 \\
Siparuna guianensis & 2 & - & 2 & 14 & 2 & 16 \\
Xylopia aromatica & 10 & 12 & 22 & 14 & 2 & 16 \\
Chaetocarpus echinocarpus & - & - & - & 11 & 1 & 12 \\
Maprounea guianensis & - & - & - & 8 & 1 & 9 \\
Matayba guianensis & 5 & 0 & 5 & 7 & 2 & 9 \\
Cordiera sessilis & 2 & - & 2 & 6 & - & 6 \\
Myrcia splendens & 6 & 1 & 7 & 5 & 1 & 6 \\
Erythroxylum daphnites & 4 & 3 & 7 & 5 & - & 5 \\
Aspidosperma multiflorum & 4 & 1 & 5 & 3 & - & 3 \\
Guapira graciliflora & 19 & 7 & 26 & 3 & - & 3 \\
Antonia ovata & 1 & 5 & 6 & 1 & - & 1 \\
Eriotheca gracilipes & 5 & 1 & 9 & 1 & - & 1 \\
Guapira noxia & 8 & 4 & 12 & - & 1 & 1 \\
Roupala montana & 8 & 10 & 18 & 1 & - & 1 \\
Syagrus flexuosa & 3 & 6 & 9 & 1 & - & 1 \\
Annona coriacea & 3 & 3 & 6 & - & - & - \\
\hline & & & & & & \\
\hline
\end{tabular}

the studied savanna forest, cause gap openings, accelerating community dynamics and contributing to the maintenance of $T$. vulgaris and other species that demand similar light levels to establish and grow. In the present study, the increase in density of T. vulgaris between 2002 and 2008 is characterized by the ingression of juvenile individuals; and the increase in basal area, in addition to the juveniles that entered the community, was due to the fast growth of adults that still remained in the community: Miguel et al. (2011) recorded the highest absolute value of periodic annual increment $(2.05 \mathrm{~cm} /$ year $)$ for this species. Therefore, differently from what was observed in tropical forests subjected to abiotic environmental changes, such as the case of riparian forests submitted to the seasonal flood of rivers and to an intense edge effect (Felfili 1993; Miguel
\& Marimon 2008), the temporal changes recorded in the savanna forest studied may have a biotic origin, led by Tachigali vulgaris, which might be a keystone species in the dynamics of this savanna forest. Besides, considering that this species maintained itself in the same hierarchical position during the study period, possibly its adult and senile individuals, after falling, opened new gaps, maintaining the possibility of regeneration and growth of the species in a type of positive feedback or virtual circle (Miguel et al. 2011).

Based on this assumption, the contribution of Tachigali vulgaris may be important to several ecosystem processes that affect community structure, as for instance the microclimate, since microclimatic factors such as light, humidity and soil and air temperature depend on canopy characteristics, especially regarding the dynamics 
of gap formation (Guilherme 2000). In this case, forests with a highly dynamic canopy, as in the present study, undergo high levels of intermediate disturbance during a short period of time, revealing a selection of tree species that are best adapted to such environmental conditions, which could be considered, hence, best competitors (Lopes \& Schiavini 2007). Therefore, changes in environmental conditions, on which Tachigali vulgaris seems to have an effective participation, are causing changes in the floristic composition and in the structure of the savanna forest under study.

Considering the ten most important species, only the first three in decreasing order (Hirtella glandulosa, Tachigali vulgaris and Xylopia aromatica) kept their IV position unchanged in 2002, 2005 and 2008 (Tab. 1; Marimon-Junior \& Haridasan 2005). It is important to highlight that those species also exhibited the highest basal area values, occurred in higher frequency compared to others and were the only ones that exhibited relative density over 5\%. Between 2002 and 2005, based on the study of Marimon-Junior \& Haridasan (2005), nearly all species changed their hierarchical IV positions. This pattern of structural change in most species gives this savanna forest a very dynamic character compared to other Cerrado vegetation types (Felfili et al. 2000; Marimon 2005; Miguel \& Marimon 2008). In this context, Baker et al. (2004) and Wright (2005) observed that changes in structure and species composition of tropical forests may have important implications in the carbon cycle and in the biodiversity of these forests.

Considering the ten most important species during the three periods of sampling, it was observed that Eriotheca gracilipes occupied the $4^{\text {th }}$ IV position in the first inventory (2002), moved to the $9^{\text {th }}$ position in 2005 and to the $12^{\text {th }}$ position in 2008 . Another remarkable change was recorded in the hierarchical position of Roupala montana, which moved from the $7^{\text {th }}$ position in 2002 for the $10^{\text {th }}$ position in 2005 and the $15^{\text {th }}$ position in 2008 . In this case, the species mentioned above, which are heliophilous $(E$. gracilipes) and pioneer (R. montana) (Franczak 2009), may have been affected by a possible partial closure of the canopy in the last period of the study (2005 to 2008). Whereas Chaetocarpus echinocarpus, which is a typical understory species (shady environments) from seasonal semideciduous and riparian forests of eastern Mato Grosso (Marimon et al. 2001; 2002), climbed from the $11^{\text {th }}$ position in 2005 to the $7^{\text {th }}$ position in 2008.
Ronquim et al. (2003) observed that Eriotheca gracilipes needs high solar radiation levels for growth (100\% transmittance), under which it exhibits higher photosynthetic capacity and higher biomass accumulation. According to these authors, under shaded conditions (30\% transmittance) E. gracilipes does not produce enough resources to sustain the demand required for the formation of reproductive structures. In this case, under natural conditions it would tolerate shading, but would remain in a vegetative state with reduced growth. According to Mendonça et al. (2008), Roupala montana is a species that occurs mainly in cerrado sensu stricto, "campo sujo", "campo de murundus" and rocky savanna (cerrado rupestre). Felfili \& Abreu (1999) recorded higher growth of $R$. montana under higher light conditions. Therefore, considering the ecological characteristics mentioned for these species and taking into account the changes in their hierarchical positions in the present study, it is suggested that the savanna forest studied here is closing.

The changes in IV in the community indicate that most species alternate frequently, as recorded by Carvalho (1992) and Felfili (1993) in Amazonian and riparian forests in central Brazil, indicating that these communities are in a dynamic state, varying in density and basal area of species over time.

Tree species such as Tachigali vulgaris may contribute not only to the understanding of complex ecological interactions of tropical forests, but also to studies on restoration of degraded areas. The restoration processes, which are usually difficult and long under the climatic conditions of central-western Brazil, require the indication of species that have the same success of establishment and growth in restoration areas that they have under natural conditions.

\section{Acknowledgements}

Programa de Pós-Graduação em Ciências Florestais e Ambientais of the Universidade Federal de Mato Grosso (UFMT), Universidade do Estado de Mato Grosso (UNEMAT) - Campus de Nova Xavantina provided us with logistic support. CNPq (Project PELD-Transição Cerrado-Floresta Amazônica: bases ecológicas e sócio-ambientais para a conservação, Proc. $\mathrm{N}^{\circ} 558069 / 2009-6$ ) provided us with financial support and CAPES granted the first author a scholarship. 


\section{References}

Ackerly, D.D.; Thomas, W.W.; Ferreira, C.A.C. \& Pirani, J.R. 1989. The forest-cerrado transition zone in southern Amazonia: results of the 1985 Projeto Flora Amazônica expedition to Mato Grosso. Brittonia 41: 113-128.

Andrade, L.A.Z.; Felfili, J.M. \& Violatti, L. 2002. Fitossociologia de uma área de cerrado denso na RECOR-IBGE, Brasília-DF. Acta Botanica Brasilica 16: 225-240.

APG III - Angiosperm Phylogeny Group. 2009. An update of the angiosperm Phylogeny group classification for the orders and families of flowering plants: APG III. Botanical Journal of the Linnean Society 161: 105-121.

Aquino, F.G.; Walter, B.M.T. \& Ribeiro, J.F. 2007. Woody community dynamics in two fragments of "cerrado" stricto sensu over a seven-year period (1995-2002), MA, Brazil. Revista Brasileira de Botânica 30: 113-121.

Baker, T.R.; Phillips, O.L.; Malhi, Y.; Almeida, S.; Arroyo, L.; DiFiore, A.; Erwin, T.; Higuchi, N.; Killeen, T.J.; Laurance, S.G.; Laurance, W.F.; Lewis, S.L.; Monteagudo, A.; Neill, D.A.; Vargas, P.N.; Pitman, N.C.A.; Silva, N.M. \& Martínez, R.V. 2004. Increasing biomass in Amazonian forest plots. Philosophical Transactions of the Royal Society B 359: 353-365.

Carpentieri, A. 2008. O Portal do Roncador - História de região do Roncador e de Nova Xavantina-MT. SEC-MT.

Carvalho, J.O.P. 1992. Structure and dynamics of a logged over Brazilian Amazonian rain forest. PhD Thesis. Univerty of Oxford, Oxford. 215p.

Chagas, R.K.; Oliveira-Filho, A.T.; Van Den Berg, E. \& Scolforo, J.R.S. 2001. Dinâmica de populações arbóreas em um fragmento de Floresta Estacional Semidecidual Montana em Lavras, Minas Gerais. Revista Árvore 25: 39-57.

Condit, R.; Hubbell, S.P. \& Foster, R.B. 1995. Mortality rates of 205 Neotropical tree and shrub species and the impact of a severe drought. Ecological Monographs 65: 419-439.

Costa, A.A. \& Araújo, G.M. 2001. Comparação da vegetação arbórea de cerradão e de cerrado na Reserva do Panga, Uberlândia, Minas Gerais. Acta Botanica Brasilica 15: 63-72.

Durigan, G.; Baitello, J.B.; Franco, G.A.D.C. \& Siqueira, M.F. 2004. Plantas do cerrado paulista. Páginas \& Letras, São Paulo. 475 p.

Eiten, G. 1979. Formas fisionômicas do cerrado. Revista Brasileira de Botânica 2: 139-148.

Felfili, J. M. 1993. Structure and dynamics of a gallery forest in central Brazil. PhD. Thesis. Oxford Forestry Institute, Oxford. 180p.

Felfili, J.M. 1995. Diversity, structure and dynamics of a gallery forest in central Brazil. Vegetatio 117: 1-15.
Felfili, J.M. \& Abreu, H.A.M. 1999. Regeneração natural de Roupala montana Aubl., Piptocapha macropoda Back. e Persea fusca Mez. em quatro condições ambientais em mata de galeria na mata de galeria do Gama-D.F. Cerne 6: 125-132.

Felfili, J.M.; Hilgbert, L.F.; Franco, A.C.; Sousa-Silva, J.C.; Rezende, A.V. \& Nogueira, M.V.P. 1999. Comportamento de plântulas de Sclerolobium paniculatum Vog. var. rubiginosum (Tul.) Benth. sob diferentes níveis de sombreamento, em viveiro. Revista Brasileira de Botânica 22: 297-301.

Felfili, J.M.; Rezende, A.V.; Silva-Júnior, M.C. \& Silva, M.A. 2000. Changes in the floristic composition of cerrado sensu stricto in Brazil over a nine-year period. Journal of Tropical Ecology 16: 579-590.

Forzza, R.C.; Leitman, P.M.; Costa, A.F.; Carvalho Jr., A.A.; Peixoto, A.L.; Walter, B.M.T.; Bicudo, C.; Zappi, D.; Costa, D.P.; Lleras, E.; Martinelli, G.; Lima, H.C.; Prado, J.; Stehmann, J.R.; Baumgratz, J.F.A.; Pirani, J.R.; Sylvestre, L.; Maia, L.C.; Lohmann, L.G.; Queiroz, L.P.; Silveira, M.; Coelho, M.N.; Mamede, M.C.; Bastos, M.N.C.; Morim, M.P.; Barbosa, M.R.; Menezes, M.; Hopkins, M.; Secco, R.; Cavalcanti, T.B. \& Souza, V.C. 2010. Lista de espécies da flora do Brasil. Jardim Botânico do Rio de Janeiro. Disponível em <http://floradobrasil.jbrj.gov.br/2010/>. Acesso em 19 set 2010.

Franczak, D.D. 2009. Dinâmica da comunidade arbustiva-árborea de um cerradão e um cerrado sensu stricto no Parque do Bacaba, Nova Xavantina-MT. Dissertação de Mestrado. Universidade Federal de Mato Grosso, Cuiabá, 72p.

Gomes, B.Z.; Martins, F.R. \& Tamashiro, J.Y. 2004. Estrutura do cerradão e da transição entre cerradão e floresta paludícola num fragmento da International Paper do Brasil Ltda., em Brotas, SP. Revista Brasileira de Botânica 27: 249-262.

Guilherme, F.A.G. 2000. Efeitos da cobertura de dossel na densidade e estatura de gramíneas e da regeneração natural de plantas lenhosas em mata de galeria, Brasília-DF. Cerne 6: 60-66.

Guilherme, F.A.G. \& Nakajima, J.N. 2007. Estrutura da vegetação arbórea de um remanescente ecotonal urbano floresta-savana no Parque do Sabiá, em Uberlândia, MG. Revista Árvore 31: 329-338.

Hallé, F.; Oldeman, R.A.A. \& Tomlinson, P.B. 1978. Tropical trees and forests. Springer-Verlag, Berlin. $483 \mathrm{p}$.

Higuchi, P.; Oliveira-Filho, A.T.; Silva, A.C.; Machado, E.L.M.; Santos, R.M. \& Pifano, D.S. 2008. Dinâmica da comunidade arbórea em um fragmento de floresta estacional semidecidual montana em Lavras, Minas Gerais, em diferentes classes de solos. Revista Árvore 32: 417-426.

IBGE. 2002. Árvores do Brasil Central. Instituto Brasileiro de Geografia e Estatística, Rio de Janeiro. 417p. 
IBGE. 2004. Mapa da vegetação do Brasil. Ministério do Planejamento, Orçamento e Gestão - MAPA Diretoria de Geociências, Brasília.

Ivanauskas, N.M; Monteiro, R. \& Rodrigues, R.R. 2004. Composição florística de trechos florestais na borda Sul-Amazônica. Acta Amazonica 34: 399-413.

Kunz, S.H.; Ivanauskas, N.M. \& Martins, S.V. 2009. Estrutura fitossociológica de uma área de cerradão em Canarana, estado do Mato Grosso, Brasil. Acta Scientiarum 31: 255-261.

Ledru, M.P.; Soares-Braga, P.; Soubiès, F.; Fournier, M.; Martin, L.; Suguio, K. \& Turcq, B. 1996. The last 50000 years in the Neotropics (Southern Brazil): evolution of vegetation and climate. Palaeogeography, Palaeoclimatology, Palaeocology 123: 239-257.

Lewis, S.L.; Lopez-Gonzalez, Sonké, G.B.; AffumBaffoe, K.; Baker, T.R.; Ojo, L.O.; Phillips, O.L.; Reitsma, J.M.; White, L.; Comiskey, J.A.; Djuikouo, M.; Ewango, C.E.N.; Feldpausch, T.R.; Hamilton, A.C.; Gloor, M.; Hart, T.; Hladik, A.; Lloyd, J.; Lovett, J.C.; Makana, J.R.; Malhi, Y.; Mbago, F.M.; Ndangalasi, H.J.; Peacock, J.; Peh, K.S.H. Sheil, D.; Sunderland, T.; Swaine, M.D.; Taplin, J.; Taylor, D.; Thomas, S.C.; Votere, R. \& Wöll, H. 2009. Increasing carbon storage in intact African tropical forests. Nature 457: 1003-1007.

Lieberman, D.; Lieberman, M.; Peralta, R. \& Hartshorn, S. 1985. Mortality patterns and stand turnover rates in a wet tropical forest in Costa Rica. Journal of Ecology 73: 915-924.

Lopes, S.F. \& Schiavini, I. 2007. Dinâmica da comunidade arbórea de mata de galeria da Estação Ecológica do Panga, Minas Gerais, Brasil. Acta Botanica Brasilica 21: 249-261.

Marimon, B.S. 2005. Dinâmica de uma floresta monodominante de Brosimum rubescens Taub. e comparação com uma floresta mista em Nova Xavantina-MT. Tese Doutorado. Universidade de Brasília, Brasília. 232p.

Marimon, B.S.; Felfili, J.M.; Lima, E.S. \& Rodrigues, A.J. 2001. Distribuição de circunferências e alturas em três porções da mata de galeria do Córrego Bacaba, Nova Xavantina-MT. Revista Árvore 25: 335-343.

Marimon, B.S.; Felfili, J.M. \& Lima, E.S. 2002. Floristic and phytosociology of the gallery forest of the Bacaba Stream, Nova Xavantina, Mato Grosso, Brasil. Edinburgh Journal of Botany 59: 303-318.

Marimon, B.S. \& Lima, E.S. 2001. Caracterização fitofisionômica e levantamento florístico preliminar no Pantanal dos Rios Mortes-Araguaia, Cocalinho, Mato Grosso, Brasil. Acta Botanica Brasilica 15: 213-229.

Marimon, B.S.; Lima, E.S.; Duarte, T.G.; Chieregatto, L.C. \& Ratter, J.A. 2006. Observations on the vegetation of northeastern Mato Grosso, Brazil. IV. An analysis of the Cerrado-Amazonian Forest ecotone. Edinburgh Journal of Botany 63: 323-341.
Marimon-Junior, B.H. \& Haridasan, M. 2005. Comparação da vegetação arbórea e características edáficas de um cerradão e um cerrado sensu stricto em áreas adjacentes sobre solo distrófico no leste de Mato Grosso, Brasil. Acta Botanica Brasilica 1: 913-926.

Mendonça, R.C.; Felfili, J.M.; Walter, B.M.; SilvaJúnior, M.C.; Rezende, A.V.; Filgueiras, T.S.; Nogueira, P.E. \& Fagg, C.W. 2008. Flora vascular do bioma Cerrado: checklist com 12.356 espécies. In: Sano, S.M.; Almeida, S.P. \& Ribeiro, J.F. (eds.). Cerrado: ecologia e flora. Embrapa Informação Tecnológica, Brasília. Pp. 421-1181.

Miguel, A. \& Marimon, B.S. 2008. Mudanças na composição florística e na diversidade de espécies em três áreas da mata de galeria do Córrego Bacaba (Nova Xavantina, MT). In: Santos, J.E. \& Galbiati, C. (orgs.). Gestão e educação ambiental - água, biodiversidade e cultura. Ed. Rima, São Carlos. Pp. 93-116.

Miguel, A.; Marimon, B.S.; Oliveira, E.A.; Maracahipes, L. \& Marimon-Junior, B.H. 2011. Dinâmica da comunidade lenhosa de uma floresta de galeria na transição Cerrado-Floresta Amazônica no leste de Mato Grosso, em um período de sete anos (1999 a 2006). Biota Neotropica 11. Disponível em <http://www.biotaneotropica.org.br/v11n1/pt/ abstract?article+ bn01111012011>. Acesso em jan 2011.

Mittermeier, R.A.; Gil, P.R.; Hoffman, M.; Pilgrim, J.; Brooks, T.; Mittermeier, C.G.; Lamoreaux, J. \& Fonseca, G.A.B. 2005. Hotspots revisited: earth's biologically richest and most endangered terrestrial ecoregions. Conservation International, New York. 291p.

Müeller-Dombois, D. \& Ellenberg, H. 1974. Aims and methods of vegetation ecology. Wiley \& Sons, New York. 574p.

Nogueira, E.M.; Fearnside, P.M.; Nelson, B.W.; Barbosa, R.I. \& Keizer, E.W.H. 2008. Estimates of forest biomass in the Brazilian Amazon: new allometric equations and adjustments to biomassfrom wood-volume inventories. Forest Ecology and Management 256: 1853-1857.

Oliveira, A.P. \& Felfili, J.M. 2008. Dinâmica da comunidade arbórea de uma mata de galeria do Brasil Central em um período de 19 anos (1985-2004). Revista Brasileira de Botânica 31: 597-610.

Oliveira, E.C.L. \& Felfili, J.M. 2005. Estrutura e dinâmica da regeneração natural de uma mata de galeria no Distrito Federal, Brasil. Acta Botanica Brasilica 19: 801-811.

Oliveira, P.S. \& Marquis, R.J. 2002. The cerrados of Brazil ecology and natural history of a neotropical Savanna. Columbia University Press, New York. 398p.

Oliveira-Filho, A.T. \& Ratter, J.A. 1995. A study of the origin of Central Brazilian forests by the analysis of plant species distribution patterns. Edinburgh Journal of Botany 52: 141-194.

Oliveira-Filho, A.T.; Vilela, E.A.; Carvalho, D.D. \& Gavilanes, M.L. 1994. Effects of soils and 
topography on the distribution of tree species in a tropical riverine forest in south-eastern Brazil. Journal of Tropical Ecolgy 10: 483-508.

Oliveira-Filho, A.T.; Mello, J.M. \& Scolforo, J.R. 1997. Effects of past disturbance and edges on tree community structure and dynamics within a fragment tropical semideciduous forest in south-eastern Brazil over a fiveyear period (1987-1992). Plant Ecology 131: 45-66.

Pereira-Silva, E.F.L.; Santos, J.E.; Kageyama, P.Y. \& Hardt, E. 2004. Florística e fitossociologia dos estratos arbustivo e arbóreo de um remanescente de cerradão em uma Unidade de Conservação do estado de São Paulo. Revista Brasileira de Botânica 27: 533-544.

Phillips, O.L.; Malhi, Y.; Vincenti, B.; Baker, T.; Lewis, S.L.; Higuchi, N.; Laurance, W.F.; Vargas, P.N.; Martinez, R.V.; Laurance, S.; Ferreira, L.V.; Stern, M.; Brown, S. \& Grace, J. 2002. Changes in growth of tropical forests: evaluating potential biases. Ecological Applications 12: 576-587.

Pott, A. \& Pott, V.J. 1994. Plantas do Pantanal. EMBRAPA-CPAP/SPI, Brasília. 320p.

Ratter, J.A. 1971. Some notes on two types of cerradão occurring in northeastern Mato Grosso. In: Ferri, M.G. (ed.). III Simpósio Sobre o Cerrado. EDUSP/ Edgard Blücher, São Paulo. Pp. 110-112.

Ratter, J.A.; Richards, P.W.; Argent, G. \& Gifford, D.R. 1973. Observations on the vegetation of the northeastern Mato Grosso. I. The woody vegetation types of the Xavantina-Cachimbo Expedition area. Philosophical Transactions of the Royal Society of London B 266: 449-492.

Rizzini, C.T. 1979. Tratado de fitogeografia do Brasil: aspectos sociológicos e florísticos. EDUSP/ HUCITEC, São Paulo. 374p.
Ronquim, C.S.; Prado, C.H.A. \& Paula, N.F. 2003. Growth and photosynthetic capacity in two woody species of Cerrado vegetation under different radiation availability. Brazilian Archieves of Biology and Technology 46: 243-252.

Sheil, D.; Burslem, D.F.R.P. \& Alder, D. 1995. The interpretation of mortality rates measures. Journal of Tropical Ecology 83: 331-333.

Sheil, D.; Jennings, S. \& Savill, P. 2000. Long-term permanent plot observations of vegetation dynamics in Budongo, a Ugandan rain forest. Journal of Tropical Ecology 16: 765-800.

Shepherd, G.J. 1994. FITOPAC 1 - Manual do Usuário. UNICAMP, Campinas. 88p.

Silva, M.R. \& Araújo, G.M. 2009. Dinâmica da comunidade arbórea de uma floresta semidecidual em Uberlândia, MG, Brasil. Acta Botanica Brasilica 23: 49-56.

Silva, F.A.M.; Assad, E.D. \& Evangelista, B.A. 2008. Caracterização climática do bioma Cerrado. In: Sano, S.M.; Almeida, S.P. \& Ribeiro, J.F. (eds.). Cerrado: ecologia e flora. Embrapa Informação Tecnológica, Brasília. Pp. 69-88.

Swaine, M.D.; Lieberman, D. \& Putz, F.E. 1987. The dynamics of tree populations in tropical forests: a review. Journal of Tropical Ecology 3: 359-366.

Werneck, M.S.; Franceschinelli, E.V. \& Tameirão-Neto, E. 2000. Mudanças na florística e estrutura de uma floresta decídua durante um período de quatro anos (1994-1998), na região do Triângulo Mineiro, MG. Revista Brasileira de Botânica 23: 401-413.

Wright, S.J. 2005. Tropical forests in a changing environment. Trends in Ecology and Evolution 20: 553-560.

Zar, J.H. 1999. Biostatistical analysis. Prentice Hall, New Jersey. 663p. 